\title{
Uncertainty Propagation in Hypersonic Aerothermoelastic Analysis
}

\author{
Nicolas Lamorte, $\stackrel{*}{*}$ Peretz P. Friedmann, $\_$and Bryan Glaz \\ University of Michigan, Ann Arbor, Michigan 48109-2140 \\ and \\ Adam J. Culler, $\underline{\S}$ Andrew R. Crowell, $\mathbb{I}$ and Jack J. McNamara** \\ Ohio State University, Columbus, Ohio 43210-1226 \\ DOI: $10.2514 / 1 . C 032233$
}

\begin{abstract}
A framework for uncertainty propagation in hypersonic aeroelastic and aerothermoelastic analyses is presented. First, the aeroelastic stability of a typical section representative of a control surface on a hypersonic vehicle is examined. Variability in the uncoupled natural frequencies of the system is modeled using beta probability distributions. Uncertainty in the flutter Mach number is computed using stochastic collocation. Next, the stability of an aerodynamically heated panel representing a component of the skin of a hypersonic vehicle is considered. In this case, uncertainty is due to the location of transition from laminar to turbulent flow and the heat flux prediction. The effect of propagating these uncertainties on vehicle behavior is determined. For both cases, uncertainty is treated using stochastic collocation, which is a new and effective approach for incorporating uncertainty in this class of problems.
\end{abstract}

\begin{tabular}{|c|c|c|}
\hline & & Nomenclature \\
\hline$A_{j}$ & $=$ & fitting coefficients \\
\hline$A, B$ & $=$ & coefficients for beta distribution \\
\hline$A_{n}(t)$ & $=$ & deformed shape coefficients \\
\hline$a$ & $=$ & normalized elastic axis location, positive af \\
\hline$b$ & $=$ & $c / 2$ semichord \\
\hline$C_{1}, \ldots, C_{4}$ & $=$ & deformed shape coefficients \\
\hline$c$ & $=$ & chord \\
\hline$D_{1}$ & $=$ & bending stiffness of the panel \\
\hline$\hat{f}(\xi)$ & $=$ & $\begin{array}{l}\text { polynomial response surface of the output } \\
\text { of interest }\end{array}$ \\
\hline$H$ & $=$ & altitude \\
\hline$h$ & $=$ & plunge degree of freedom \\
\hline$h_{p}$ & $=$ & panel thickness \\
\hline$h_{1}$ & $=$ & radiation shield thickness \\
\hline$h_{2}$ & $=$ & thermal insulation thickness \\
\hline$I_{\alpha}$ & $=$ & $\begin{array}{l}\text { static moment of inertia of the wing section } \\
\text { about elastic axis }\end{array}$ \\
\hline$K_{h}$ & $=$ & spring constant in plunge \\
\hline$K_{\alpha}$ & $=$ & spring constant in pitch \\
\hline$k$ & $=$ & thermal conductivity \\
\hline$L$ & $=$ & lift \\
\hline$l_{p}$ & $=$ & panel length \\
\hline
\end{tabular}

Presented as Paper 2010-2964 at the 51st AIAA/ASME/ASCE/AHS/ASC Structures, Structural Dynamics, and Materials Conference, Orlando, FL, 12 15 April 2010; received 11 December 2012; revision received 17 June 2013; accepted for publication 25 June 2013; published online 22 January 2014. Copyright $\odot 2013$ by the authors. Published by the American Institute of Aeronautics and Astronautics, Inc., with permission. Copies of this paper may be made for personal or internal use, on condition that the copier pay the $\$ 10.00$ per-copy fee to the Copyright Clearance Center, Inc., 222 Rosewood Drive, Danvers, MA 01923; include the code 1542-3868/14 and $\$ 10.00$ in correspondence with the CCC.

*Ph.D. Candidate, Department of Aerospace Engineering. Student Member AIAA.

${ }^{\dagger}$ François-Xavier Bagnoud Professor, Department of Aerospace Engineering. Fellow AIAA.

${ }^{\ddagger}$ Postdoctoral Researcher, Department of Aerospace Engineering; currently Research Aerospace Engineer, Vehicle Technology Directorate, U.S. Army Research Laboratory, Aberdeen Proving Ground, Maryland 21005. Senior Member AIAA.

${ }^{\S}$ Ph.D. Candidate, Department of Aerospace Engineering; currently Program Manager, Sierra Lobo, Inc., U.S. Air Force Research Laboratory, Wright-Patterson Air Force Base, OH. Member AIAA.

${ }^{\top} \mathrm{Ph} . \mathrm{D}$. Candidate, Department of Aerospace Engineering. Student Member AIAA.

**Associate Professor, Department of Aerospace Engineering. Senior Member AIAA.

\begin{tabular}{|c|c|c|}
\hline$M_{e a}$ & $=$ & aerodynamic moment \\
\hline$M_{f}$ & $=$ & flutter Mach number \\
\hline$M_{f d}$ & $=$ & deterministic flutter Mach number \\
\hline$M_{T}$ & $=$ & thermal bending moment \\
\hline$M_{\infty}$ & $=$ & freestream Mach number \\
\hline$m$ & $=$ & cross-sectional mass of the cross section \\
\hline$m_{f} \equiv\langle f\rangle$ & $=$ & mean of $f$ \\
\hline$N_{I}$ & $=$ & $\begin{array}{l}\text { number of points in the numerical } \\
\text { integration scheme }\end{array}$ \\
\hline$N_{v}$ & $=$ & number of random variables \\
\hline$N_{x}$ & $=$ & in-plane stress resultant \\
\hline$P(x, t)$ & $=$ & pressure \\
\hline$P+1$ & $=$ & number of interpolating function \\
\hline $\begin{array}{l}P_{3} \\
p(\xi)\end{array}$ & & $\begin{array}{l}\text { pressure behind the leading-edge shock } \\
\text { probability density function }\end{array}$ \\
\hline$p_{f}(\beta)$ & $=$ & probability of failure \\
\hline$p_{(A, B)}(\xi)$ & $=$ & beta $(A, B)$ probability density function \\
\hline$Q_{\text {aero }}$ & $=$ & aerodynamic heat flux \\
\hline$Q_{\mathrm{rad}}$ & $=$ & radiation heat flux \\
\hline$q_{a}$ & $=$ & $P(x, t)-P_{3}$, aerodynamic pressure on the panel \\
\hline$r_{\alpha}$ & $=$ & nondimensional radius of gyration \\
\hline$S_{\alpha}$ & $=$ & $\begin{array}{l}\text { static mass moment of the wing section } \\
\text { about elastic axis }\end{array}$ \\
\hline$T$ & $=$ & temperature \\
\hline$T_{0}$ & $=$ & initial panel temperature \\
\hline$T_{\text {wall }}$ & $=$ & wall temperature \\
\hline$T_{f}$ & $=$ & flight Time \\
\hline$t$ & $=$ & time \\
\hline$t_{h}$ & $=$ & $\tau b$, airfoil thickness \\
\hline$U_{\infty}$ & $=$ & freestream velocity \\
\hline$v_{n}$ & $=$ & velocity of the airfoil surface in the $z$ direction \\
\hline$w(x, t)$ & $=$ & out-of-plane panel displacement \\
\hline$w_{k}$ & $=$ & numerical integration scheme weights \\
\hline$x$ & $=$ & coordinate on the panel \\
\hline$x_{e}$ & $=$ & distance of panel location from leading edge \\
\hline$x_{t i}$ & $=$ & $\begin{array}{l}\text { location of transition point from laminar to } \\
\text { turbulent flow from leading edge }\end{array}$ \\
\hline$x_{\alpha}$ & $=$ & normalized center of gravity location, positive aft \\
\hline$y=f(x)$ & $=$ & output of interest \\
\hline$Z_{s}(x, y)$ & $=$ & structural shape \\
\hline$\alpha$ & $=$ & pitch degree of freedom \\
\hline$\alpha_{q}$ & $=$ & aerodynamic heat flux scaling factor \\
\hline$\alpha_{s}$ & $=$ & static angle of attack \\
\hline$\beta$ & $=$ & $\frac{M_{f}}{M_{f d}}$, flutter Mach number ratio \\
\hline$\beta_{s}$ & $=$ & shock angle \\
\hline$\Gamma$ & $=$ & gamma function \\
\hline
\end{tabular}




$\begin{array}{lll}\gamma & = & \text { specific heat ratio } \\ \Delta T_{f} & = & T_{f}-T_{f d}, \text { variation of flight time } \\ \delta_{j k} & = & \text { Kronecker symbol } \\ \theta & = & \text { forebody surface inclination } \\ -1 \leq \xi \leq 1 & = & \text { normalized random variable associated } \\ & & \text { with uncertain input } \\ \mu_{L} & = & \text { laminar coefficient of viscosity } \\ \mu_{T} & = & \text { turbulent coefficient of viscosity } \\ \xi & = & \text { vector of uncertain inputs } \\ \xi_{k} & = & \text { numerical integration scheme points } \\ \langle\xi\rangle & = & \text { mean of } \xi \\ \rho_{p} & = & \text { density of the panel } \\ \sigma_{f} & = & \text { standard deviation of } f \\ \tau & = & \text { interpolating polynomials functions } \\ \phi_{i}(\xi) & =\sqrt{K_{h} / m}, \text { plunge natural frequency } \\ \omega_{h} & =\sqrt{K_{\alpha} / I_{\alpha}}, \text { pitch natural frequency } \\ \omega_{\alpha} & \end{array}$

\section{Introduction}

$\mathbf{H}$ YPERSONIC flight is an active area of research motivated by interest in unmanned rapid response to threats and reusable launch vehicles for affordable access to space [1-6]. Such vehicles are based on lifting-body designs, which tightly integrate the airframe and propulsion system. For hypersonic cruise applications, the propulsion system is expected to consist of air-breathing engines that operate for sustained periods in atmospheric flight $[5,7,8]$. Flying at hypersonic speeds within the atmosphere causes severe aerodynamic heating. Accurate modeling of the resulting aerothermoelastic interactions is important for hypersonic vehicle performance, stability, and reliability analyses.

Hypersonic flows are inherently complex and involve phenomena that are not present in supersonic conditions such as dissociations, chemically reacting flow, viscous interactions, and higher levels of aerodynamic heat flux $[7,8]$. There are no suitable high-speed, highenthalpy tunnels capable of testing scaled models of hypersonic vehicles. Furthermore, hypersonic aerothermoelastic scaling laws are not available at high Mach numbers [9]. Therefore, the development of accurate computational aerothermoelastic simulation capabilities is critical for the design and analysis of hypersonic vehicles.

High-fidelity numerical simulations of the complex hypersonic flow environment are computationally expensive, and the state of art is at a relatively early stage of development where the precise role of important factors such as real gas effects, chemically reacting flows, and complex viscous interactions are not understood. Current analysis tools employ computationally efficient models based on simplifying assumptions of the physics and/or reduced-order modeling (ROM) of full-order computations. Compensating for these shortcomings in modeling requires the use of uncertainty propagation techniques in hypersonic aerothermoelastic analyses.

Two common simplifying assumptions used in aerothermoelastic analysis of hypersonic vehicles are piston theory for computing the aerodynamic loading [1] and Eckert's reference temperature or enthalpy method for calculating aerodynamic heat flux [7]. Use of these assumptions requires the introduction of uncertainty because several important effects are neglected. Similarly, the use of ROMs currently under development for a hypersonic vehicle [10-15] introduces additional sources of error. Thus, the uncertainty due to unmodeled physics as well as the approximation errors associated with ROMs have to be modeled. Several approaches for propagating uncertainty in aeroelastic problems have been considered, such as direct Monte Carlo simulation (MCS) [16], polynomial chaos expansion (PCE) [17], and adaptive finite elements [18]. Stochastic collocation (SC), which is employed in the present study, is an effective alternative to direct Monte Carlo simulation, which requires prohibitive computational costs for complex problems. Furthermore, SC does not require modifications to deterministic analyses codes (i.e., it is nonintrusive) and was shown to outperform PCE in a recent study [19].
The overall objective of this paper is to investigate the effects of several uncertainty sources relevant to hypersonic vehicle design. The approach used for propagating the uncertainty is stochastic collocation [19], which has never been used for hypersonic aeroelasticity or aerothermoelasticity.

1) The aeroelastic stability of a typical section representative of a control surface on a hypersonic vehicle is investigated first. In this case, uncertainty is associated with the natural bending and torsional frequencies, and SC is used to quantify the effect of uncertainty on the flutter Mach number.

2) The aerothermoelastic stability of a panel located on a vehicle flying at hypersonic speeds is considered next. For this case, uncertainties due to modeling assumptions associated with the aerodynamic heat flux and laminar to turbulent transition predictions are quantified, and their effects on flight time before the onset of panel flutter is examined.

\section{Uncertainty Propagation}

Once the outputs of interest have been defined and the uncertain inputs have been identified, probabilistic approaches can be used to quantify uncertainty effects. In this study, the effects of uncertain inputs $\xi$ are propagated through a computational analysis symbolically represented by $f$ to quantify uncertainty effects on the output of interest $f(\xi)$. The uncertainty propagation analysis is illustrated in Fig. 1. The function $f$ represents the aeroelastic or the aerothermoelastic stability analyses described in Secs. III.A.1, III.B.1, where $\xi$ are the uncertain input parameters. The probabilistic approach to uncertainty quantification consists of the following steps.

1) Each uncertain input is treated as a random variable characterized by a probability distribution $p(\xi)$.

2) SC is used to approximate the computationally expensive functional dependance of the output of interest on the uncertain inputs, i.e., $f(\xi)$ is approximated.

3) Conventional MCS methods are applied to the computationally efficient approximate representation obtained from SC. The effects of the uncertain inputs on the output of interest are quantified in terms of probability distributions denoted by $p(y)$.

Detailed descriptions of the probabilistic characterizations of the uncertain inputs and the SC function approximations are provided next.

\section{A. Characterization of Input Probability Distribution}

Variability associated with an uncertain input is modeled by a probability density function (PDF) $p(\xi)$, and $p\left(\xi_{0}\right) d \xi$ is the probability that $\xi_{0}-d \xi / 2 \leq \xi \leq \xi_{0}+d \xi / 2$. Thus, the PDF $p\left(\xi_{0}\right)$ describes the probability of occurrence that the random variable $\xi$ will have the value of $\xi_{0}$. Commonly used PDFs include normal, lognormal, exponential, or Cauchy distributions, which are defined on unbounded domains. Using such PDFs may require evaluating the output of interest at input combinations with no physical significance and/or leading to unfeasible computations. In contrast, beta distributions, given in Eq. (1), represent a family of bounded probability distributions in which the range of the random input variables can be controlled by prescribing bounds. Moreover, the choice of the two parameters $A$ and $B$ permits one to control the PDF shape as illustrated in Fig. 2. The parameters $A$ and $B$ control the shape of the tail of the PDF at the bounds $\xi=-1$ and $\xi=1$, respectively. The values $A=1$ and $B=1$ yield a nonzero PDF at the two edges. A value for $A, B$ greater than 1 produces a flat tail as illustrated in Fig. 2. Thus, high values of $A$ and $B$ produce a PDF that assigns small probabilities to uncertain parameters at the outer edges, thus emphasizing the central portion. Thus, uniform, symmetric, or

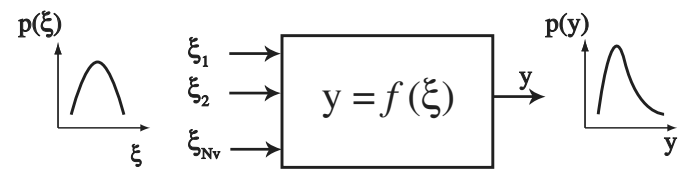

Fig. 1 Uncertainty propagation approach. 


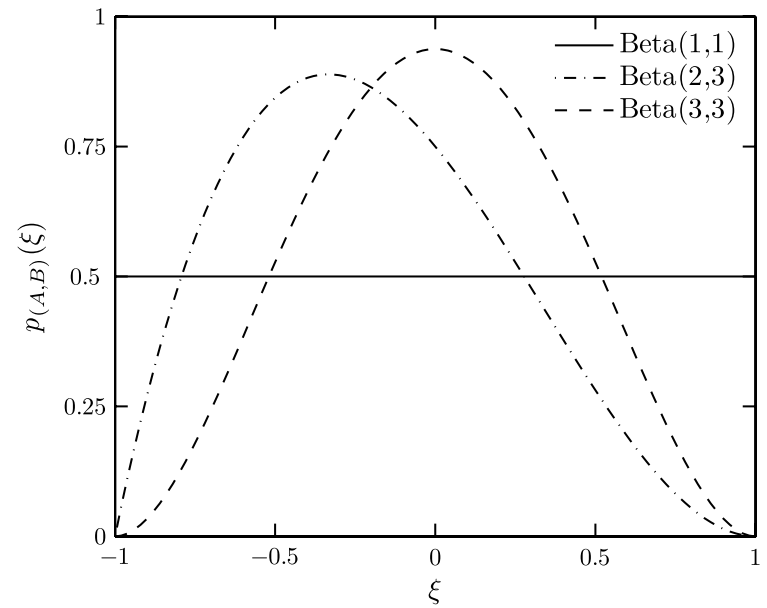

Fig. 2 Examples of beta PDF shapes for different combinations of $A$ and $B$.

nonsymmetric PDFs can be accommodated over the input range by using beta distributions [20]. A beta distribution corresponding to particular values of $A$ and $\bar{B}$ is denoted by $\operatorname{Beta}(A, B)$ :

$$
p_{(A, B)}(\xi)=\frac{\Gamma(A+B)}{\Gamma(A) \Gamma(B)} \frac{(1+\xi)^{A-1}(1-\xi)^{B-1}}{2^{A+B-1}}
$$

\section{B. Stochastic Collocation}

Once the sources of uncertainty are identified and quantified by appropriate probability distributions, the effects of uncertainty can be studied using two types of approaches: intrusive [18,21-23] and nonintrusive $[16,19,24]$. Hypersonic aerothermoelastic problems require the use of nonintrusive methods due to the complexity of comprehensive analysis codes.

Direct Monte Carlo simulation is the simplest nonintrusive approach used in relatively simple aeroelastic studies [16,17,25-31]. This method requires numerous evaluations of the function of interest (e.g., flutter Mach number) at values of the uncertain inputs dictated by their probability distributions. The computational cost associated with numerous analysis evaluations is prohibitive for complex problems such as hypersonic aeroelastic and aerothermoelastic analysis. Therefore, SC is employed in this study as a computationally efficient alternative to direct MCS.

In SC, computationally efficient polynomial response surfaces are used to approximate the functional relationship between uncertain inputs $\xi$ and the output of interest $f(\xi)$, where $\xi$ is a normalized random variable varying between the limits -1 and 1 :

$$
f(\xi) \approx \hat{f}(\xi)=\sum_{j=1}^{P+1} A_{j} \phi_{j}(\xi)
$$

The response surface $\hat{f}$ given by Eq. (2) consists of an expansion in terms of polynomial basis functions $\left(\phi_{j}(\xi)\right)_{1<j<P+1}$, in which $A_{j}$ are fitting coefficients, and $P+1$ represents the number of basis functions. Once constructed, MCS can be applied to the computationally inexpensive polynomial response surface to obtain the probability distribution associated with the output of interest.

In the current study, the expensive analyses are evaluated at a set of inputs $\xi$, called collocation points. The collocation points are chosen such that mean $m_{f}$, given by Eq. (3), and variance $\sigma_{f}^{2}$, given by Eq. (4), are estimated using a numerical integration scheme defined by $N_{I}$ integration points, $\left(\xi_{k}\right)_{k=1, N_{I}}$ and their corresponding weights $\left(w_{k}\right)_{k=1, N_{I}}$. Thus, the collocation points correspond to the numerical integration points:

$$
m_{f}=\int_{\Omega} p(\xi) f(\xi) \mathrm{d} \xi \simeq \sum_{k=1}^{N_{I}} w_{k} f\left(\xi_{k}\right)
$$

$$
\sigma_{f}^{2}=\int_{\Omega} p(\xi)(f(\xi)-\langle f\rangle)^{2} \mathrm{~d} \xi \simeq \sum_{k=1}^{N_{I}} w_{k}\left(f\left(\xi_{k}\right)-\langle f\rangle\right)^{2}
$$

For the one-dimensional case, the polynomial response surface, given by Eq. (2), is generated using Lagrange polynomials $\left(\phi_{j}\right)_{j=1, P+1}$ [Eq. ()] associated with the collocations points $\left(\xi_{k}\right)_{k=1, N_{I}}$ [Eq. ( $\left.\left.\underline{6}\right)\right]$ :

$$
\begin{gathered}
\phi_{j}(\xi)=\prod_{k=1, k \neq j}^{N_{I}} \frac{\xi-\xi_{k}}{\xi_{j}-\xi_{k}} \quad j=1, \quad P+1 \\
\phi_{j}\left(\xi_{k}\right)=\delta_{j k} \quad k=1, N_{I} \quad j=1, \quad P+1
\end{gathered}
$$

The degree of the polynomial approximation $P$ in Eqs. (5) and ( $\underline{6})$ is equal to $N_{I}-1$.

For a multidimensional random input space, $\boldsymbol{\xi}=\left(\xi^{i_{v}}\right)_{i_{v}=1, N_{v}}$, in which $N_{v}$ is the number of uncertain inputs, the multivariate extension of Eq. (5) is given by Eq. (7):

$$
\phi_{j}(\boldsymbol{\xi})=\prod_{i_{v}=1}^{N_{v}} \prod_{k=1, k \neq j}^{N_{I}} \frac{\xi^{i_{v}}-\xi_{k}^{i_{v}}}{\xi_{j}^{i_{v}}-\xi_{k}^{i_{v}}} \quad j=1, \quad P+1
$$

For beta distributions, the corresponding numerical integration scheme is computed using Gaussian quadrature [32]. For a single random variable, the numerical integrations points are the roots of the Legendre polynomial function of degree $N_{I}$ associated with the beta probability distribution of the input. The numerical integration scheme is exact for polynomial functions of order less than $2 N_{I}-1$. In the two-dimensional case, the collocation points are depicted in Fig. 3 for beta distributions corresponding to various combinations of $A$ and $B$ for $N_{I}=7^{2}$. This method tends to concentrate collocation

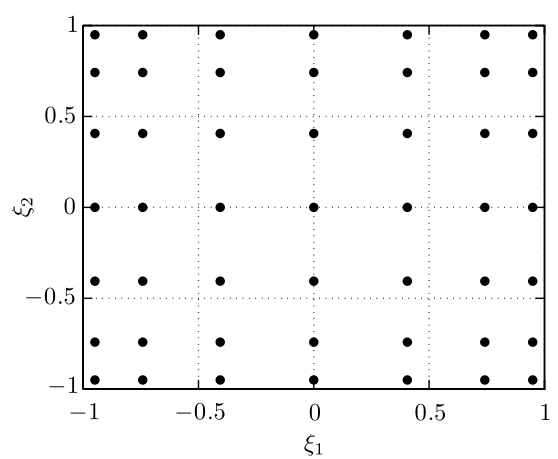

a) $\operatorname{Beta}(1,1)$

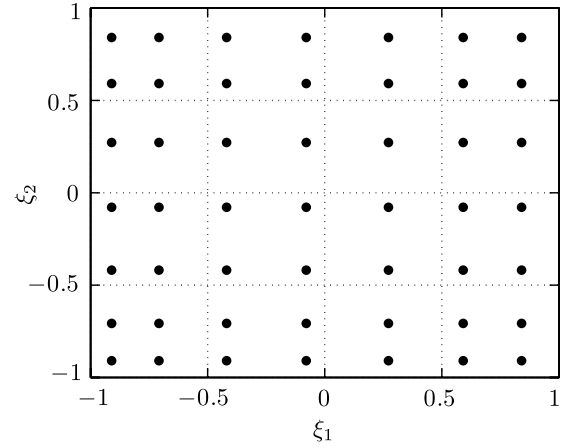

b) Beta $(2,3)$

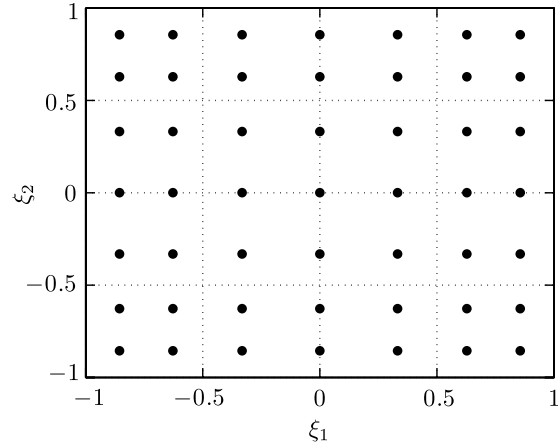

c) $\operatorname{Beta}(3,3)$

Fig. 3 Collocation points for two random variables for different beta distributions, $N_{I}=7^{2}$. 
points in the regions of higher probability. For instance, integration points associated with the $\operatorname{Beta}(3,3)$ PDF have a higher concentration at the center of the domain compared to the grid associated with $\operatorname{Beta}(1,1)$.

Because there is ample evidence that the $\mathrm{SC}$ approach is superior to polynomial chaos expansion [19] (another widely used technique), SC was selected for this study. Note, however, that this method suffers from the curse of dimensionality, which implies that increasing the number of random inputs exponentially increases the number of analyses and the computational cost of the method. The number of analyses required for the implementation of the SC approach is $(P+1)^{N_{v}}$. Furthermore, the collocation points associated with most integration schemes are located strictly within the domain of the input variable. Therefore, extrapolation is required for response surface evaluations close to the domain boundaries, which may adversely affect accuracy. Other efficient interpolation techniques can be considered to create the response surface such as adaptive sparse grid interpolation [33,34], Kriging surrogates [35], or multivariable splines [36,37] if discontinuities are present. The investigation of such approaches is beyond the scope of this paper.

\section{Results}

Two representative case studies are considered. The first corresponds to the aeroelastic analysis of a hypersonic control surface section. Next, the aerothermoelastic stability of a panel located on the surface of a hypersonic vehicle is examined.

The importance of nondeterministic approaches for hypersonic vehicle aeroelastic analysis is characterized by comparing the results with those obtained from deterministic analyses. In all results, the 95\% confidence interval for the flutter Mach number and minimum and maximum values are obtained by conducting $10^{5}$ Monte Carlo simulations on the approximate problem (i.e., the polynomial response surface) using the MATLAB random number generator for beta distributions betarnd.

\section{A. Aeroelastic Stability of a Two-Dimensional Typical Section}

\section{Description of the Aeroelastic Problem}

The flutter Mach number associated with a double wedge typical section representative of a control surface of a hypersonic vehicle is examined first. The typical section, shown in Fig. 4, is characterized by pitch $(\alpha)$ and plunge $(h)$ degrees of freedom. This problem was treated deterministically in [2].

For this particular problem, the equations of motion given in Eq. (8) can be obtained using Lagrange's equations assuming small deflections and no structural damping:

$$
\left[\begin{array}{cc}
m & S_{\alpha} \\
S_{\alpha} & I_{\alpha}
\end{array}\right]\left\{\begin{array}{l}
\ddot{h} \\
\ddot{\alpha}
\end{array}\right\}+\left[\begin{array}{cc}
K_{h} & 0 \\
0 & K_{\alpha}
\end{array}\right]\left\{\begin{array}{l}
h \\
\alpha
\end{array}\right\}=\left\{\begin{array}{c}
-L \\
M_{e a}
\end{array}\right\}
$$

The aerodynamic loading is obtained from third-order piston theory given by Eq. (9), where $v_{n}$ is the velocity normal to the chord line of the airfoil given in Eq. (10) and $Z_{s}$ accounts for the thickness of the airfoil and $w$ for the deformation:

$$
\frac{P(x, t)-P_{\infty}}{P_{\infty}}=\gamma\left[\frac{v_{n}}{a_{\infty}}+\frac{\gamma+1}{4}\left(\frac{v_{n}}{a_{\infty}}\right)^{2}+\frac{\gamma+1}{12}\left(\frac{v_{n}}{a_{\infty}}\right)^{3}\right]
$$

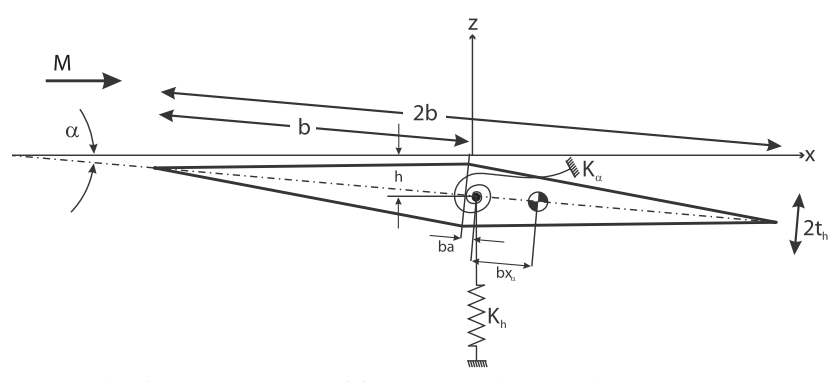

Fig. 4 Two-degree-of-freedom typical section geometry.
Table 1 Baseline configuration for the typical section

\begin{tabular}{cc}
\hline \hline Parameter & Value \\
\hline$H$ & {$\left[\begin{array}{lc}0 & 100,000 \mathrm{ft}\end{array}\right]$} \\
$a$ & {$\left[\begin{array}{cc}-0.4 & 0.4\end{array}\right]$} \\
$c$ & $2.35 \mathrm{~m}$ \\
$\tau$ & $3.36 \%$ \\
$x_{\alpha}$ & 0.2 \\
$\omega_{h}$ & $13.4 \mathrm{~Hz}$ \\
$\omega_{\alpha}$ & $37.6 \mathrm{~Hz}$ \\
\hline \hline
\end{tabular}

Table 2 Uncertain parameters associated

\begin{tabular}{|c|c|c|}
\hline Parameter & Range & Distribution \\
\hline$\omega_{h}$ & {$\left[\begin{array}{ll}-3 \% & +14 \%\end{array}\right]$} & Uniform \\
\hline$\omega_{\alpha}$ & {$\left[\begin{array}{ll}-30 \% & +0 \%\end{array}\right]$} & Uniform \\
\hline
\end{tabular}
with the two-dimensional typical section

$$
v_{n}(x ; w, \dot{w})=\frac{\partial w}{\partial t}+U_{\infty}\left[\frac{\partial}{\partial x}\left(Z_{s}+w\right)+\alpha_{s}\right]
$$

As the freestream Mach number increases, flutter ensues, and the flutter Mach number $M_{f}$ corresponds to zero damping in one of the two modes of the aeroelastic system. The output of interest is the flutter Mach number $M_{f}$. In the probabilistic approach, the probability that the flutter Mach number is less than some percentage of the deterministic prediction as given by Eq. (11) is quantified. In this example, the value of $\beta=85 \%$ is assumed to be an acceptable limit for the flutter Mach number; it corresponds to an aeroelastic stability margin of $15 \%$ :

$$
p_{f}(\beta)=p\left(M_{f}<\beta M_{f d}\right)
$$

The variability is introduced in the uncoupled natural frequencies $\omega_{\alpha}$ and $\omega_{h}$ to account for uncertainty from possible changes in frequencies due to aerodynamic heating. Therefore, the output of interest for this problem is a function of two uncertain inputs, denoted $\xi_{1}$ and $\xi_{2}$, which correspond to the natural frequencies. The aerodynamic loading is obtained from third-order piston theory. The aeroelastic stability is determined using an efficient damping identification method known as the ARMA model [38]. Damping is extracted from the transient response of the system. As dynamic pressure increases, flutter ensues, and the flutter Mach number $M_{f}$ corresponds to zero damping in one of the two modes of the system. The flutter Mach number $M_{f}$ depends also on the offset $a$ between the elastic axis and the midchord.

\section{Uncertainty Propagation for the Two-Dimensional Typical Section}

The system is represented by the parameters given in Table 1 , taken from [2]. As indicated in [39], changes in frequencies can be also considered to be representative of the combined effects of material degradation and thermal stresses that occur in a heated structure. The bending frequency increases as the structure heats up, and the torsional frequency decreases [39]. The range of variation of the bending and torsional frequencies observed for typical trajectories of a airbreathing hypersonic vehicle are $[-3,+14 \%]$ and $[-30,+0 \%]$, respectively [39], and these were selected as representative values. The respective probability distribution is chosen to be uniform as summarized in Table 2 .

Selection of the degree of the polynomial required for the SC expansion is based on a convergence study. In the convergence study, $a$ and $H$ are fixed at 0.0 and $40,000 \mathrm{ft}$, respectively. The mean and the variance associated with the flutter Mach number obtained from polynomial expansion up to 10th order are compared to results obtained from a 15th-order expansion based on $(15+1)^{2}=256$ analysis evaluations. The relative errors associated with the mean and standard deviation are shown in Fig. 5. Furthermore, the accuracy of 


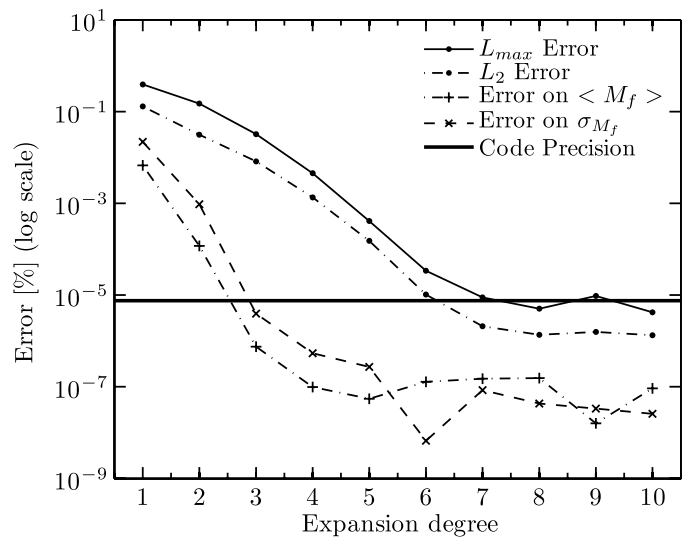

Fig. 5 Convergence study for SC method; $a=0$, and $H=40,000 \mathrm{ft}$.

the various polynomial expansions is quantified by comparing the response surface predictions with the exact values obtained for a 16 by 16 Cartesian uniform grid spanning the uncertain variables space. For the corresponding 256-point set, the maximum and sum of squares (L2) relative errors between the computed value and the response surface prediction is computed and shown in Fig. 5. The maximum error is close to the expected accuracy of the analysis for a response surface based on a sixth-order expansion (i.e., 49 collocation points). Similar convergence is observed for all of the combinations of altitude and elastic axis offset considered in this study. Therefore, the sixth-order polynomial expansions are used in generating the results corresponding to various values for the elastic axis offset $a$ and the altitude $H$.

Note that certain parameters associated with the analysis had to be modified to obtain reliable results when propagating uncertainty; the time step is divided by 2 , and the simulated time is increased by a factor of 5 compared to that used in the deterministic calculations. These modifications are necessary to allow the ARMA method to identify both frequencies and damping coefficients in the transient response, particularly when $\omega_{h}$ and $\omega_{\alpha}$ are close to each other.

Uncertainties associated with the pitch and plunge natural frequencies are propagated to the flutter Mach number using a sixthorder polynomial response surface generated by stochastic collocation. The results are depicted in Figs. $\underline{6 a}$ and $6 \mathrm{~b}$ and concisely summarized in Table 3 . In both figures, the deterministic flutter Mach number, represented by a thick line and diamond symbols, is compared to the mean in flutter Mach numbers due to the uncertainties. The expected value of flutter Mach number is depicted using the cross symbols. Furthermore, an interval likely to include the flutter Mach number with $95 \%$ confidence (95\% CI) is represented by a shaded area. The dashed and dash-dotted lines corresponds to the minimum and maximum values respectively. The $15 \%$ margin in flutter Mach number is represented by the plain line with the point symbols.

In Fig. 6a, the elastic axis offset $a$ varies from -0.4 to 0.4 , and the altitude is constant and equal to $40,000 \mathrm{ft}$. The results show that the maximum of the flutter Mach number is close to the deterministic value. Recall that the baseline analysis does not correspond to the mean values of the uncertain parameters but to $\xi_{1}=0$ and $\xi_{2}=0$, which are close to the bounds of the uncertain parameters interval. The mean of $\xi_{1}$ and $\xi_{2}$ correspond to a modification of $\omega_{h}$ and $\omega_{\alpha}$ by +11.5 and $-15 \%$, respectively. As a result of uncertainty modeling for the probabilistic case, the natural frequencies are closer to each other for $99.7 \%$ of the cases when compared to the deterministic analysis; $\omega_{h}$ increases, and $\omega_{\alpha}$ decreases. It yields a lower flutter Mach number in most cases. The standard deviation varies from 11 to $16 \%$ of the deterministic value. The $95 \%$ CIs interval varies from -61 to $+1 \%$ of the deterministic value. Similarly, the variation of the flutter Mach number with altitude is depicted in Fig. 6b, and the elastic axis offset is equal to 0.1 . Finally, in both cases, the $15 \%$ margin lies inside the interval given by the expected value and the standard deviation as well as the $95 \% \mathrm{CI}$, which corresponds to high probability of the flutter Mach number to be less than this limit.

The probability distributions of the flutter Mach number are given in Figs. 7a-7c for various elastic axis offsets $a$. The bars represents the PDF, and the curved line represents the cumulative distribution function $(\mathrm{CDF})$ of the flutter Mach number. The deterministic value and its probability are indicated on the CDF curve by a diamond symbol. The $15 \%$ margin is indicated by a the left-pointing triangle symbol. It shows that there is a significant probability that the system will flutter before the value predicted based on the baseline parameters and the margin selected. This illustrates the significance of using nondeterministic approaches to properly quantify the aeroelastic stability boundary.

Additional information can be extracted from the probabilistic nature of the uncertainty quantification analysis, as illustrated in Fig. 8a, which shows the probability that the flutter Mach number is less than some percentage of the deterministic prediction given by Eq. (11). For example, depending on the value of the elastic offset, there is a $63-78 \%$ probability that the control surface will flutter at a Mach number that is $15 \%$ less than the deterministic prediction, when assuming a PDF corresponding to $\operatorname{Beta}(1,1)$.

These results demonstrate that uncertain inputs can produce significant levels of variability in predicted flutter Mach numbers. By treating the problem in a probabilistic manner, more information about the flutter margin is extracted. In this particular case, the deterministic analysis is not sufficient because large variations in the flutter Mach number due to the assumed uncertainties are found.

The effect of the input probability distribution shape of the random inputs on the stochastic output probability distribution is also considered using the framework developed. The range for the frequencies is unchanged. Both $\xi_{1}$ and $\xi_{2}$ have the identical probability distribution for all cases shown in Figs. $\underline{8}$ and $\underline{9}$. The

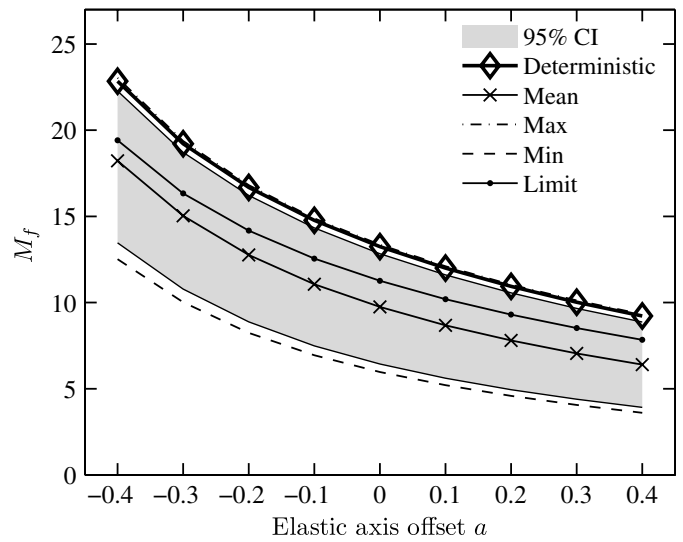

a) $H=40,000 \mathrm{ft}$

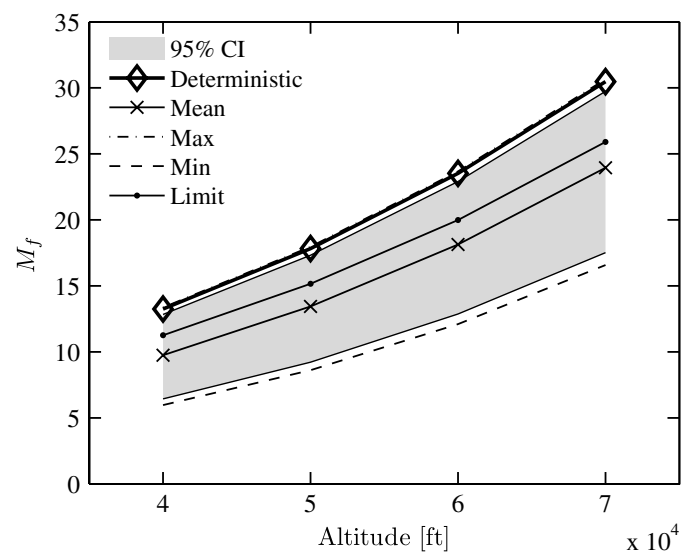

b) $a=0$

Fig. 6 Uncertainty propagation results for varying a) elastic axis, and b) altitude. 
Table 3 Flutter Mach number variability; $H=40,000 \mathrm{ft}$

\begin{tabular}{|c|c|c|c|c|c|c|c|}
\hline Elastic offset & $M_{f}$ & $\left\langle M_{f}\right\rangle$ & $\sigma_{M_{f}}$ & $95 \mathrm{CI}$ & Normalized 95 CI, \% & Range & Normalized range, $\%$ \\
\hline$a=-0.4$ & 22.84 & $18.23(-20.17 \%)$ & $2.60(11.40 \%)$ & {$[13.47,22.28]$} & {$\left[\begin{array}{ll}-41.09, & -2.48\end{array}\right]$} & {$[12.52,23.03]$} & {$[-45.18$} \\
\hline$a=0$ & 13.25 & $9.75(-26.38 \%)$ & $1.93(14.59 \%)$ & {$[6.44,12.84]$} & {$[-15.47$} & {$[5.97,13.36]$} & {$[-54.92$,} \\
\hline$a=0.4$ & 9.22 & $6.41(-30.46 \%)$ & $1.50(16.26 \%)$ & {$[3.92,8.88]$} & {$[-27.54$} & {$[3.61,9.32]$} & -60.89 \\
\hline
\end{tabular}

results illustrate the effect of the probability distribution of the input as illustrated by Figs. $8 \mathrm{~b}-8 \mathrm{~d}$ and Figs. $9 \mathrm{a}-9 \mathrm{c}$. The choice of input probability distribution affects all the stochastic analysis quantities (i.e., mean or expected value, standard deviation, $95 \%$ confidence interval, and probability of failure). As expected, maximum and minimum are not modified because the ranges of the uncertain inputs are the same. In all cases, the $\mathrm{CI}$ is reduced. This behavior is a result of the monotonic relation between the inputs and output as well as the shape of the probability distributions chosen for the inputs. All three distributions give more probability to the interior of the domain of uncertain inputs. Therefore, the CI of the uncertain inputs is smaller, which yields a smaller $\mathrm{CI}$ for the output. In addition, the $\mathrm{CI}$ of the flutter Mach number moves closer to the maximum in the case of
$\operatorname{Beta}(3,2)$ and closer to the minimum in the case of $\operatorname{Beta}(2,3)$. In fact, the $\operatorname{Beta}(2,3)$ distribution gives more probability to the lower values of the frequencies compared to $\operatorname{Beta}(3,2)$. Therefore there is more probability given to the lower Mach numbers. It shows that the statistical results should be interpreted in the context of the assumptions made regarding the uncertainty of the inputs.

When considering these results, it is important to note that the altitude $(H=40,000 \mathrm{ft})$ at which the aeroelastic studies are conducted is not realistic of hypersonic flight. However more realistic altitudes of 80,000-100,000 ft result in high Mach numbers, and therefore the altitude is artificially reduced to obtain more practical values. However, as pointed out in [2] and shown here through the uncertainty propagation approach, incorporation of aerodynamic

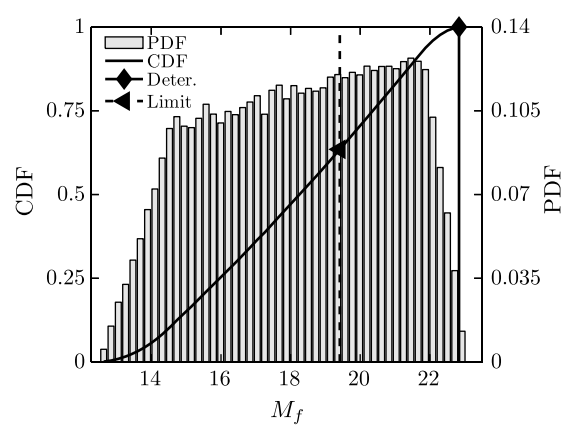

a) $a=-0.4$

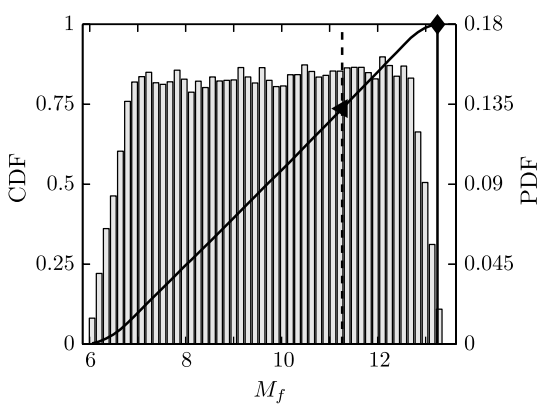

b) $\boldsymbol{a}=\mathbf{0}$

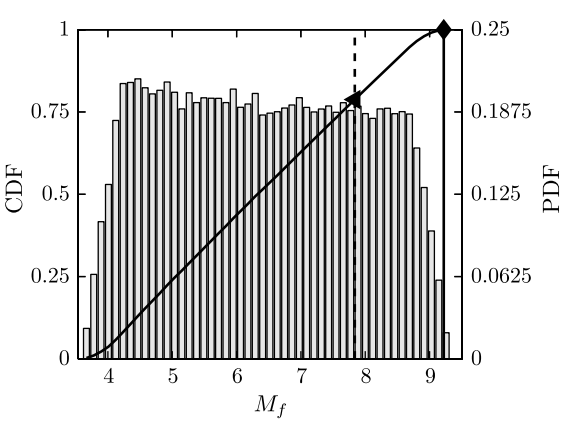

c) $a=0.4$

Fig. 7 Flutter Mach number PDF prediction using a sixth-order polynomial expansion in SC; $H=40,000$ ft.

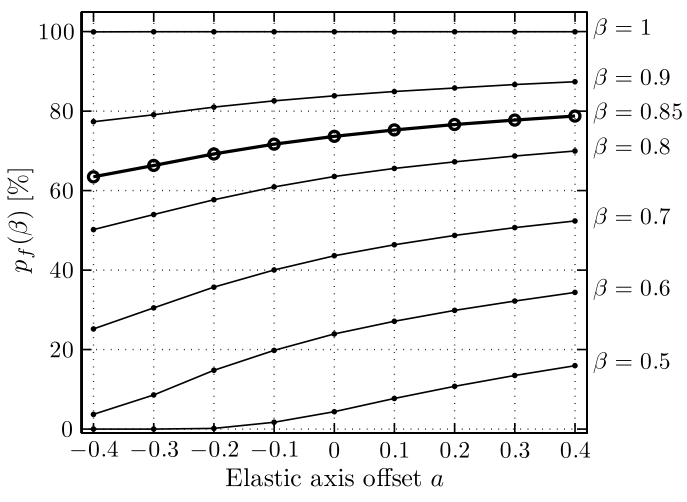

a) $\operatorname{Beta}(1,1)=$ Uniform

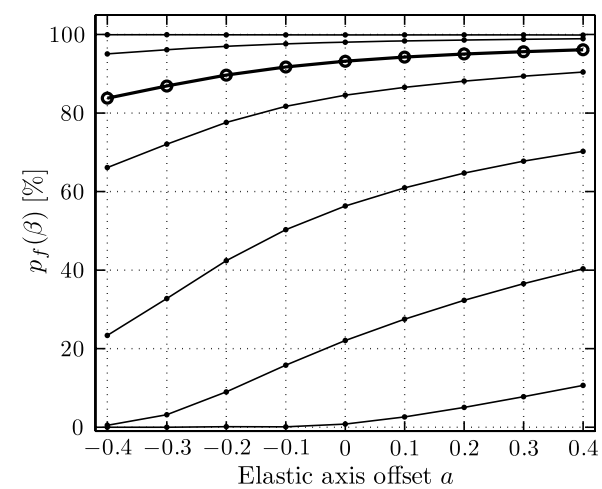

c) $\operatorname{Beta}(2,3)$

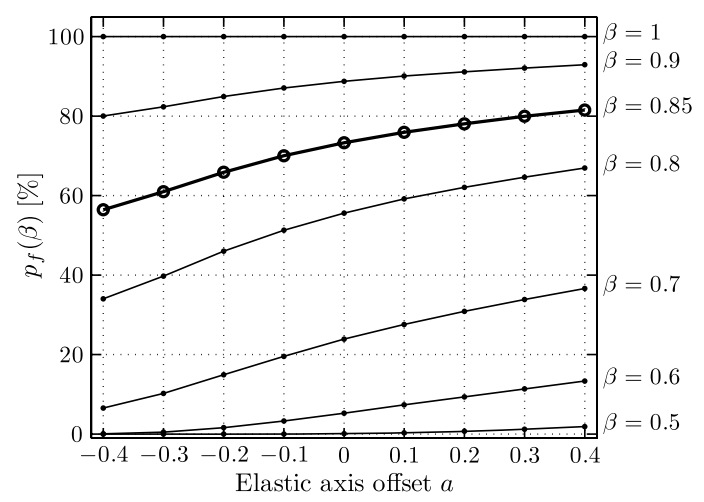

b) $\operatorname{Beta}(3,2)$

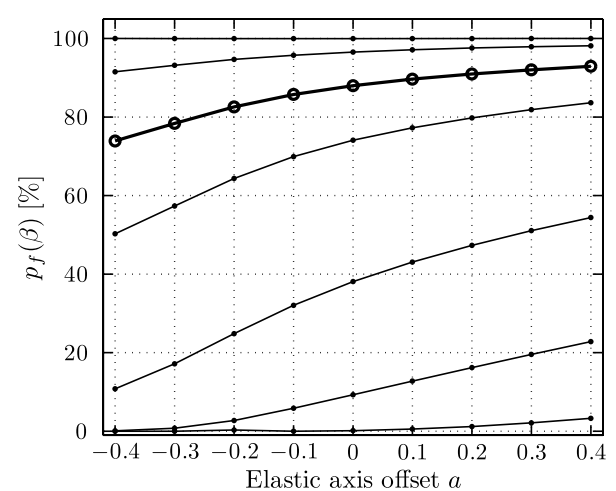

d) $\operatorname{Beta}(3,3)$

Fig. $8 p_{f}(\beta)$ for different inputs probability distributions; $H=40,000 \mathrm{ft}$. 


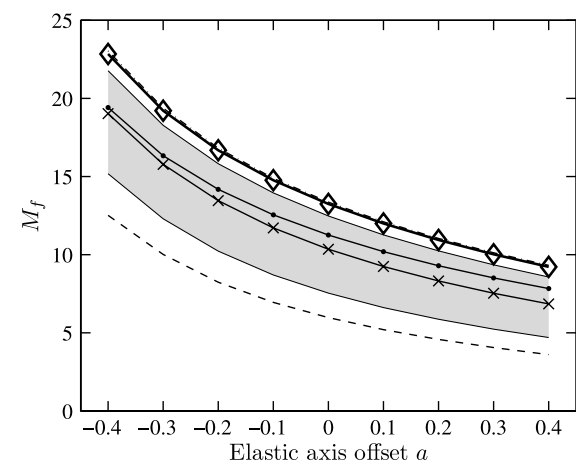

a) Beta $(3,2)$

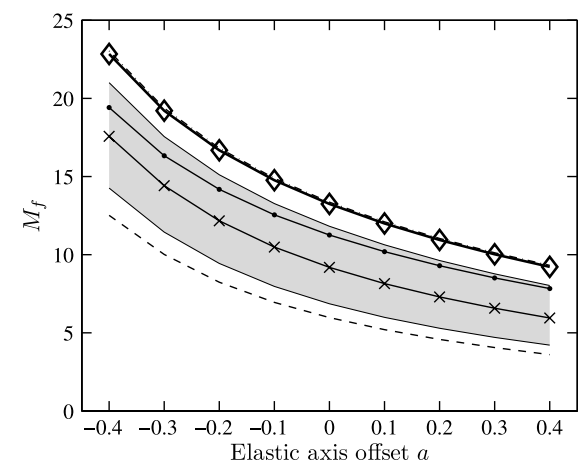

b) Beta $(2,3)$

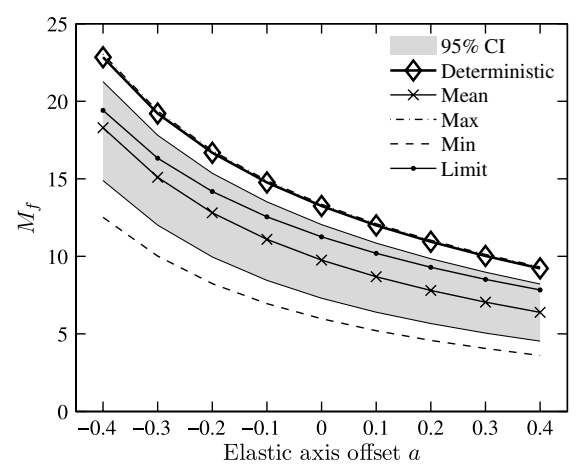

c) Beta $(3,3)$

Fig. 9 Uncertainty propagation results for different input probability distribution; $H=40,000 \mathrm{ft}$.

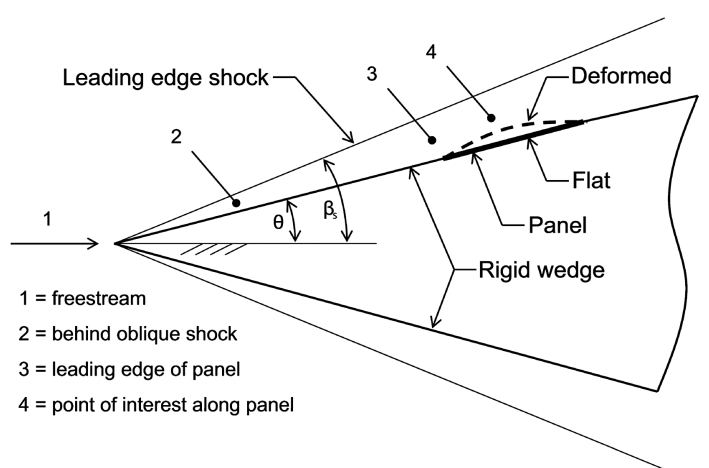

Fig. 10 Panel located on an inclined surface of a wedge-shaped forebody.

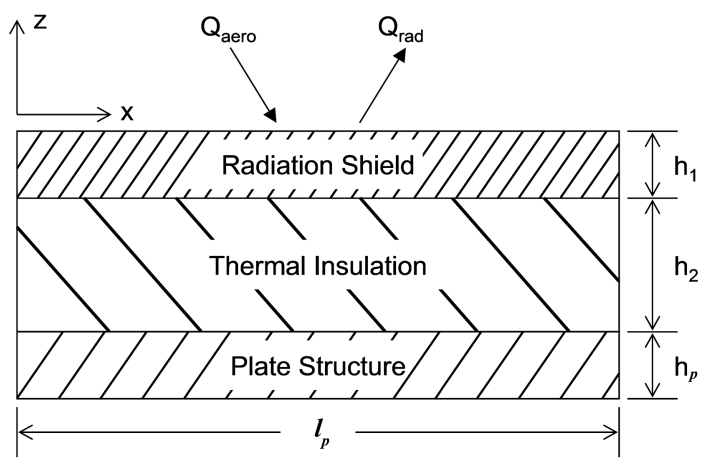

Fig. 11 Two-dimensional model of the thermal structure.

heating leads to a reduction of the flutter Mach number, and thus aerothermoelastic studies that account for the effects of heat transfer will produce operating altitudes and Mach numbers more representative of hypersonic vehicle operating regime.

\section{B. Aerothermoelastic Behavior of a Panel}

\section{Description of the Aerothermoelastic Problem}

The second problem is the aerothermoelastic stability of an aerodynamically heated two-dimensional panel. A detailed deterministic study of this problem was performed in [13]. The panel depicted in Fig. 10 is located on the surface of a vehicle flying at hypersonic speeds. It is assumed that the panel is covered by a thermal protection system consisting of a radiation shield and thermal insulation, shown in Fig. 11. The radiation shield is a PM-2000 honeycomb sandwich, and the thermal insulation is internal multiscreen insulation. The plate structure is composed of a hightemperature grade of titanium (Ti-6Al-2Sn-4Zr-2Mo). The material properties are given at $300 \mathrm{~K}$ in Table 4 . Specific heat and thermal conductivity are temperature-dependent $[\underline{13}, \underline{40}, \underline{41}]$.
The aeroelastic model for this problem is obtained by combining the two-dimensional, moderate deflection von Kármán plate theory given in Eq. (12) with unsteady aerodynamic loading $q_{a}$, based on third-order piston theory. It accounts for thermal stresses as well as change in material properties due to effect of temperature. The effects of thermal loading due to arbitrary in-plane and through-thickness temperature distributions are included in $N_{x}$ and $M_{T}$, respectively. The TPS contributes to the mass but not to the stiffness.

$$
D \frac{\partial^{4} w}{\partial x^{4}}-N_{x} \frac{\partial^{2} w}{\partial x^{2}}+\rho_{p} \frac{\partial^{2} w}{\partial t^{2}}+q_{a}+\frac{\partial^{2} M_{T}}{\partial x^{2}}=0
$$

The nonlinear equations of motion are solved using a Galerkin approach to discretize the spatial dependance, and the time-domain panel response is obtained from a fourth-order Runge-Kutta scheme. The out-of-plane displacement $w(x, t)$ [Eq. (13)] is expressed as a combination of sine modes and a third-order polynomial function uniquely defined to satisfy nonhomogeneous boundary conditions due to thermal loads:

$$
\begin{aligned}
& w(x, t)=\sum_{n=1}^{6} A_{n}(t) \sin \left(n \pi \frac{x}{l_{p}}\right)+C_{1}(t)+C_{2}(t) x+C_{3}(t) x^{2} \\
& +C_{4}(t) x^{3}
\end{aligned}
$$

The heat transfer problem is treated using Eckert's reference enthalpy model for evaluating the aerodynamic heat flux [42]. The temperature distribution in the structure is computed from a finite-difference solution of the heat transfer problem given by Eq. (14):

$$
\rho c \frac{\partial T}{\partial t}=k_{x} \frac{\partial^{2} T}{\partial x^{2}}+k_{z} \frac{\partial^{2} T}{\partial z^{2}}
$$

It is assumed that the vehicle is in straight and level flight, at a constant altitude and Mach number. Because the edges of the panel are fixed at its end points, thermal stresses develop as the panel is heated, leading to buckling and eventual aerothermoelastic instability. The through-the-thickness temperature gradients bend the panel upward before it flutters. The instant when the panel starts to flutter determines the flight time $T_{f}$ that characterizes the stability boundary of the system, and it represents the time to failure. This flight time corresponds to the instant when the out-of-plane panel displacement at the midchord point reaches $-10 \%$ of the panel thickness. This metric is chosen as a useful value for identifying the onset of flutter from the transient response of the panel. This value for

\section{Table 4 Properties of the thermal structure at $300 \mathrm{~K}$}

\begin{tabular}{lcccc}
\hline \hline & $\rho, \mathrm{kg} / \mathrm{m}^{3}$ & $c, \mathrm{~J} / \mathrm{kg} / \mathrm{K}$ & $k, \mathrm{~W} / \mathrm{m} / \mathrm{K}$ & $h_{i}, \mathrm{~mm}$ \\
\hline Radiation shield & 359 & 465 & 0.250 & 7.4 \\
Thermal insulation & 73.0 & 729 & 0.0258 & 10.0 \\
Plate structure & 4540 & 463 & 6.89 & 5.0 \\
\hline \hline
\end{tabular}


Table 5 Baseline configuration for the panel

\begin{tabular}{cc}
\hline \hline Parameter & Value \\
\hline$H$ & $98,500 \mathrm{ft}$ \\
$M_{\infty}$ & $8-12$ \\
$\theta$ & $5 \mathrm{deg}$ \\
$l_{p}$ & $1.5 \mathrm{~m}$ \\
$h_{p}$ & $5 \mathrm{~mm}$ \\
$T_{0}$ & $300 \mathrm{~K}$ \\
$x_{e}$ & $1.0 \mathrm{~m}$ \\
$x_{t i}$ & $1.0 \mathrm{~m}$ \\
\hline \hline
\end{tabular}

the out-of-plane displacement signifies the onset of oscillating values for $w$, which is indicative of panel flutter. This metric for the onset of flutter is accurate to within $\pm 1 \mathrm{~s}$ of flight time, based on the nature of the results generated.

Because aerodynamic loading, elastic deformation, inertial loads, and heat transfer are tightly coupled [13], heat flux prediction is a key component of the analysis. The use of Eckert's reference enthalpy model implies several assumptions about the modeling of the heating problem, which introduce sources of uncertainty and affect the stability of the system. Therefore, uncertainty in the heat flux prediction has to be quantified. A scaling factor $\alpha_{q}$ for the heat flux is introduced and treated as the first random variable $\xi_{1}$. The location of the transition from laminar to turbulent flow, $x_{t i}$, is treated as the second random variable $\xi_{2}$.

\section{Uncertainty Propagation for the Aerothermoelastic Problem of a Panel}

This problem is defined by the parameters provided in Table $\underline{5}$. The altitude considered is $98,500 \mathrm{ft}$, and the freestream Mach number varies between 8 and 12 . The forebody inclination is $5 \mathrm{deg}$; the panel is assumed to be $1.5 \mathrm{~m}$ long and is located at a distance of $1.0 \mathrm{~m}$ from

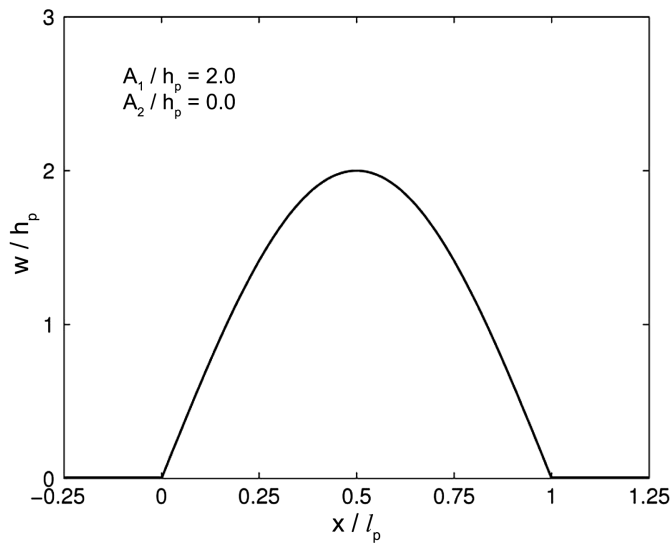

a) Deformed panel shape 1

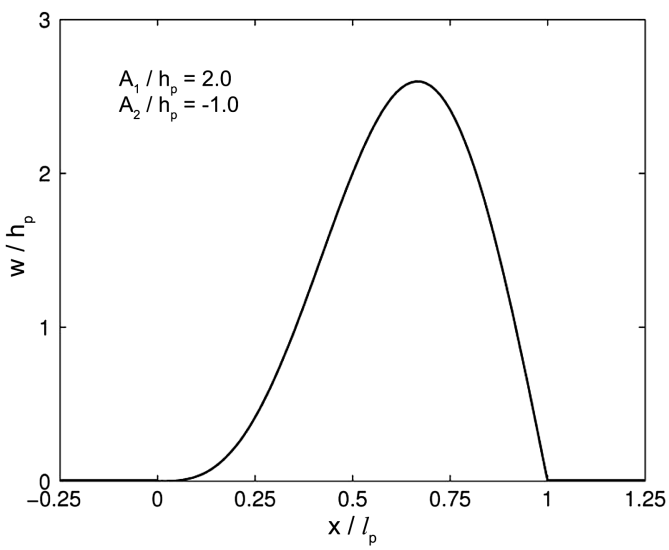

c) Deformed panel shape 2 the leading edge of the vehicle. The flow over the panel is assumed to be fully turbulent because transition is assumed to start at the leading edge of the vehicle and to end before the leading edge of the panel. Thus, in this problem, uncertainty is introduced in the heat flux transfer computations.

Appropriate modeling of turbulence and gas properties is a key factor for accurate prediction of the aerodynamic heat flux on the structure. In [13], two sources of uncertainty have been identified. The first is associated with uncertainty in turbulence modeling, and the second pertains to uncertainty in the transition from laminar to turbulent flow. Both influence the heat flux and thus have a direct impact on the aerothermoelastic stability of the panel.

a. Turbulence Modeling. The uncertainty due to turbulence modeling was quantified by comparing Eckert's reference enthalpy model [42] with full-order CFD results based on two turbulence models; this comparison is depicted in Fig. 12. The full-order results were generated with the CFL3D code [ $4 \overline{3}]$ for a flight Mach number of 8.0 and a surface temperature on the panel of $900 \mathrm{~K}$. For the panel deflections shown in Figs. 12a and 12c, the predicted heat flux distributions along the panel computed using the various models are illustrated in Figs. 12b and 12d. It is evident that the results depend on the model used to compute the convective heat flux. Four different predictions are compared: two based on CFD computations with different turbulence models, namely Menter and Wilcox available in CFL3D [43], and two based on Eckert's reference enthalpy and reference temperature models. The models considered in Fig. 12 result in similar spatial distribution shapes for the heat flux and differ only in the magnitude of the heat flux.

Based on these results, the uncertainty due to turbulence modeling is characterized by the variation in heat flux predictions based on the differences between Eckert's reference enthalpy model, and the CFD results based on different turbulence models are accounted for by using a scaling factor $\alpha_{q}$ that modifies the Eckert's reference enthalpy

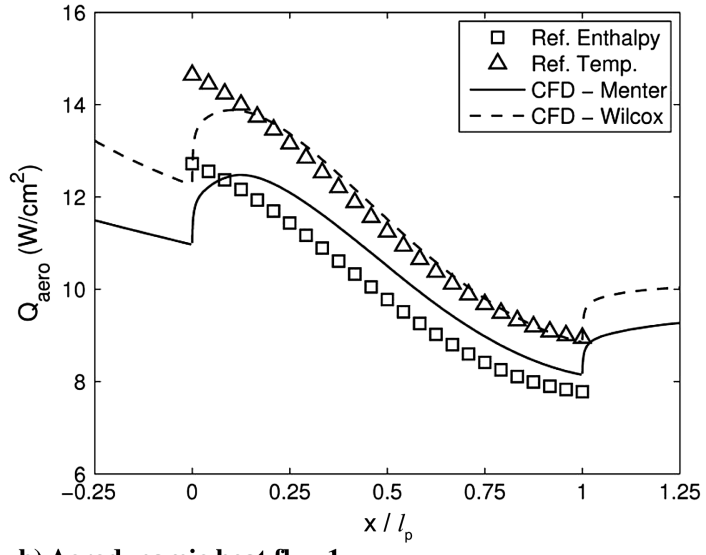

b) Aerodynamic heat flux 1

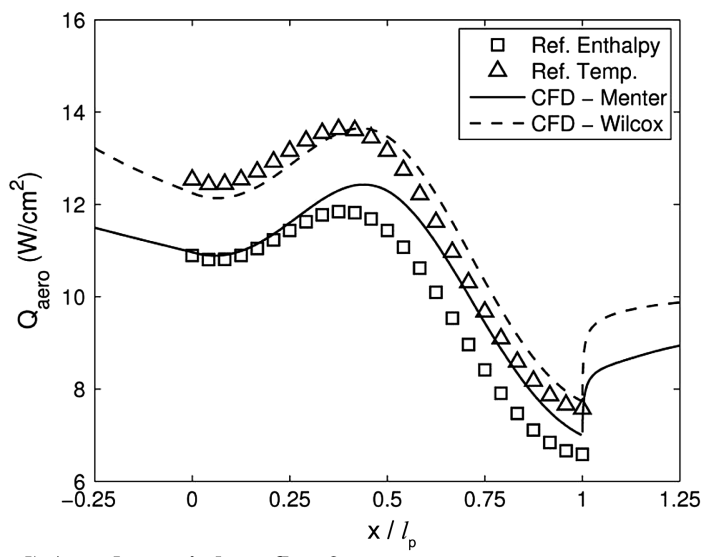

d) Aerodynamic heat flux 2

Fig. 12 Comparison of aerodynamic heating predictions over two deformed panel shapes; $T_{\text {wall }}=900 \mathrm{~K}$, and $M=8$ (from [13, $\left.\underline{14}\right]$ ). 


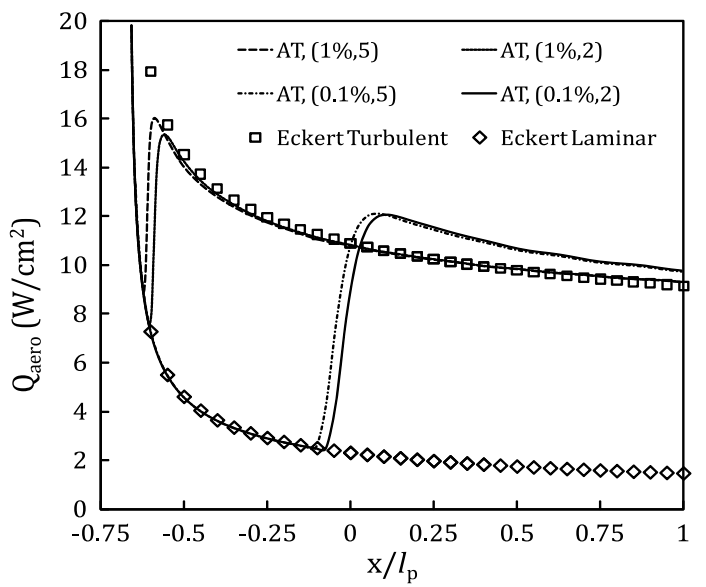

Fig. 13 Heat flux prediction using CFD++ $k-\epsilon$ augmented with AT model; $T_{\text {wall }}=900 \mathrm{~K}$, and $M=8$.

Table 6 Uncertain parameters

\begin{tabular}{cccc}
\hline \hline Parameter & Baseline value & Range & Distribution \\
\hline$\alpha_{q}$ & 1 & {$[0.951 .25]$} & Uniform \\
$x_{t i}, \mathrm{~m}$ & 1 & {$[0.21]$} & Uniform \\
\hline \hline
\end{tabular}

heat flux $Q_{\text {aero }}$ to yield $\alpha_{q} Q_{\text {aero }}$. The range for $\alpha_{q}$ is chosen to be $0.95<\alpha_{q}<1.25$ to encompass the difference between Eckert's reference enthalpy and both CFD results shown in Figs. 12b and 12d. A value of $\alpha_{q}=1$ corresponds to the baseline value employed in [14]. The probability distribution for $\alpha_{q}$ is assumed to be uniform, i.e., $\operatorname{Beta}(1,1)$.

b. Transition Location Prediction. In addition to the uncertainty associated with the turbulence model, the uncertainty associated with the location of the onset of transition is also modeled. In [13], the location at which the flow transitions from laminar to turbulent was arbitrarily selected to correspond to the distance between the leading edge of the vehicle and the leading edge of the panel. Transition modeling in hypersonic flow is a complex issue. The location of the transition region depends on numerous parameters such as flight conditions, wall temperature, surface roughness, or disturbances levels present in the flow [7].

Uncertainty in the transition is quantified by using CFD++, a commercially available computational fluid dynamics (CFD) solver. The CFD++ code contains several turbulence models. A turbulence model recommended for external hypersonic aerodynamic predictions is the $k-\epsilon$ model. To model the transition location, an algebraic transition (AT) model is used in conjunction with the $k-\epsilon$

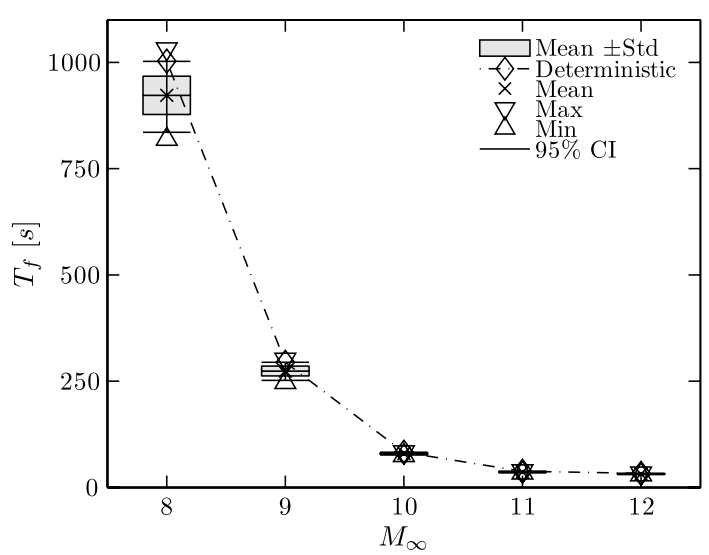

a) Flight time model [44]. It triggers transition based on detection of local flow curvature by augmenting local shear stress.

For each of the additional turbulence equations, boundary conditions are needed. The dependent variable associated with each freestream boundary condition can be computed given two freestream turbulence characteristic parameters: the turbulent kinetic energy intensity $T_{u}$ (which varies from 0.1 to $1 \%$ for external flows according to [44]) and the turbulent-to-laminar viscosity ratio $\mu_{T} / \mu_{L}$ (which varies from 2 to 5). The combination of both parameters characterizes the level of turbulence in the freestream flow. However, these parameters are rarely known [44] and therefore should be treated as uncertain parameters. To estimate their impact on the heat flux prediction that depends on laminar-to-turbulent transition region location on the vehicle, four simulations were conducted for different cases that correspond to the extreme values of both parameters. For level flight at Mach 8 and a constant wall temperature of $900 \mathrm{~K}$, the location of the turbulence transition region can be determined from the heat flux distributions shown in Fig. 13. The sharp vertical increases in heat flux indicate transition from laminar to turbulent flow. Turbulence transition location for different values of kinetic energy intensity varies from close to the leading edge of the vehicle, which corresponds to $x / l_{p}=-0.67$, up to the leading edge of the panel $\left(x / l_{p}=0\right)$.

In the deterministic analysis, it was assumed that the panel is exposed to fully developed turbulent flow. This assumption is conservative. For laminar flows, the aerodynamic heat flux on the panel is approximately one-fifth of the heat flux due to turbulent flows. However, there is uncertainty associated with the location of the transition due to variability in the turbulence level of the freestream flows. To quantify the effects of uncertainty associated with the turbulence onset location, $x_{t i}$, the distance of the onset location from the leading edge of the panel is varied from 0.2 to $1 \mathrm{~m}$, corresponding to $-0.67 \leq x_{t i} / l_{p} \leq-0.1$. The distance of $1 \mathrm{~m}$

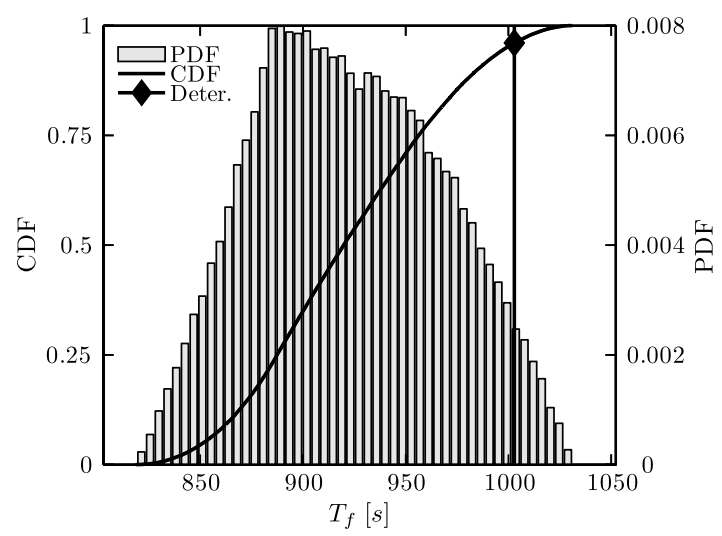

Fig. 15 PDF for the flight time at Mach 8; $H=98,500 \mathrm{ft}$.

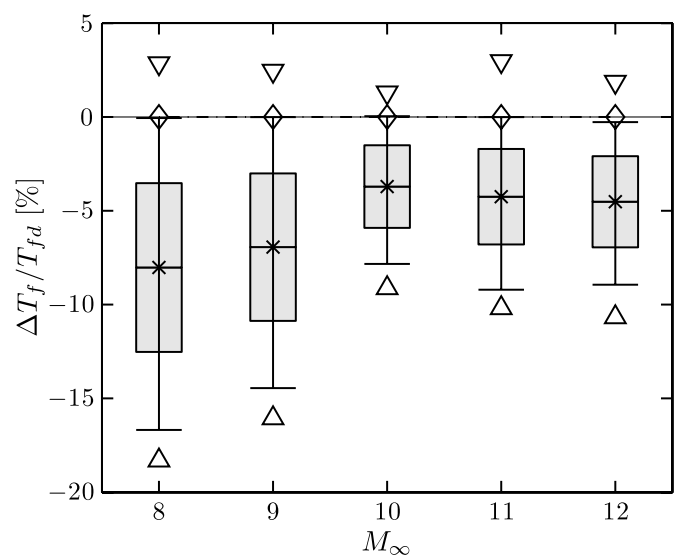

b) Normalized flight time

Fig. 14 Flight time as a function of flight Mach number. 
Table $7 \quad$ Flight time variability

\begin{tabular}{|c|c|c|c|c|c|c|c|}
\hline Mach number & $T_{f}, \mathrm{~s}$ & $\left\langle T_{f}\right\rangle, \mathrm{s}$ & $\sigma_{T_{f}}, \mathrm{~s}$ & $95 \% \mathrm{CI}, \mathrm{s}$ & Normalized $95 \mathrm{CI}, \%$ & Range, $\mathrm{s}$ & Normalized range, $\%$ \\
\hline$M_{\infty}=8$ & 1002.8 & $922.4(-8.0 \%)$ & $45.1(4.5 \%)$ & {$[842.5,1009.2]$} & {$[-16.0$} & {$[819.3,1031.5]$} & {$[-18.3,+2.9]$} \\
\hline$M_{\infty}=9$ & 294.3 & $273.9(-6.9 \%)$ & $11.6(3.9 \%)$ & {$[253.5,296.0]$} & {$[-13.9$} & {$[247.1,301.6]$} & {$\left[\begin{array}{ll}-16.0, & +2.5\end{array}\right]$} \\
\hline$M_{\infty}=10$ & 82.8 & $79.7(-3.71 \%)$ & $1.8(2.2 \%)$ & {$[76.6,83.1]$} & {$[-7.5$} & {$[75.2,83.9]$} & {$\left[\begin{array}{ll}-9.1, & +1.3]\end{array}\right.$} \\
\hline
\end{tabular}

corresponds to an onset of turbulent flow at the leading edge of the vehicle, which represents the baseline value assumed in [13]. The turbulence onset location is assumed to be uniformly distributed between 0.2 and $1 \mathrm{~m}$.

Both uncertainties associated with the turbulence model and the transition location, summarized in Table 6 , are propagated through the analysis and their impact on the flight time is determined, where flight time is used as metric for onset of instability. A sixth-order polynomial response surface is constructed based on 49 analysis runs (i.e., seven collocation points for the two random variables). Uncertainty propagation results for different Mach numbers are shown Figs. 14a and 14b. In both figures, the lines correspond to the deterministic values. The thick bars correspond to the expected values plus/minus the standard deviation, and the error bars correspond to the $95 \%$ CI. The downward and upward pointing triangles correspond to the maximum and minimum values, respectively. In Fig. 14b, the results depicted in Fig. 14a are replotted in terms of variation of flight time $\Delta T_{f}$ and normalized with respect to the deterministic value $T_{f d}$.

At Mach 8, the mean value of the flight time is $922 \mathrm{~s}$, compared to the deterministic value of $1003 \mathrm{~s}$. The standard deviation and range are $45.1 \mathrm{~s}(4.5 \%)$ and $820-1032 \mathrm{~s}([-18 \%,+3 \%])$, respectively, where percentages are in terms of the deterministic value. The results indicate that the expected flight time is less than the deterministic value, which is close to the maximum flight time at each specified Mach number. This trend is to be expected, considering the choice of the uncertain variables and their respective ranges. The range of the coefficient $\alpha_{q}$ leads to increased heat flux amplitude in $83 \%$ of the cases. When transition is located closer to the leading edge of the panel, the heat flux on the panel is also increased. These effects reduce the flight time.

Additional details can be gleaned from the probability distribution shown in Fig. 15. The bars represent the PDF and the curved line corresponds to the CDF. The deterministic value is identified by the vertical line and the diamond symbol. The output probability distribution indicates a significant probability that the flight time will be much less than its deterministic value at Mach 8 . These results illustrate the importance of incorporating uncertainty in a more complicated aerothermoelastic problem.

The same uncertainty is also propagated at different Mach numbers, and a concise summary of the probability results is presented in Table 7. The probability distributions for the additional Mach numbers are given in Fig. 16. The ranges for $\alpha_{q}$ and the transition location determined from $\bar{M}$ ach 8 results are used in these computations. The trends observed for Mach 9 and 10 depicted in Figs. $16 \mathrm{a}$ and $16 \mathrm{~b}$ are similar. However, discrepancies are observed for Mach numbers 11 and 12, as shown in Figs. 16c and $16 \mathrm{~d}$, respectively. The shapes of the PDF curves are not smooth despite lack for any physical reasons that would justify strong nonlinear relation between the uncertain inputs and output. Approximately $\pm 1 \mathrm{~s}$ errors occur in the estimations of flight time, as described in Sec. III.B.1. For lower Mach numbers, this error is insignificant compared to the magnitude of the deterministic value and the stochastic variability of the output. However, for higher Mach numbers, this error grows and becomes significant compared to the estimated flight times and significatively affects the response surface
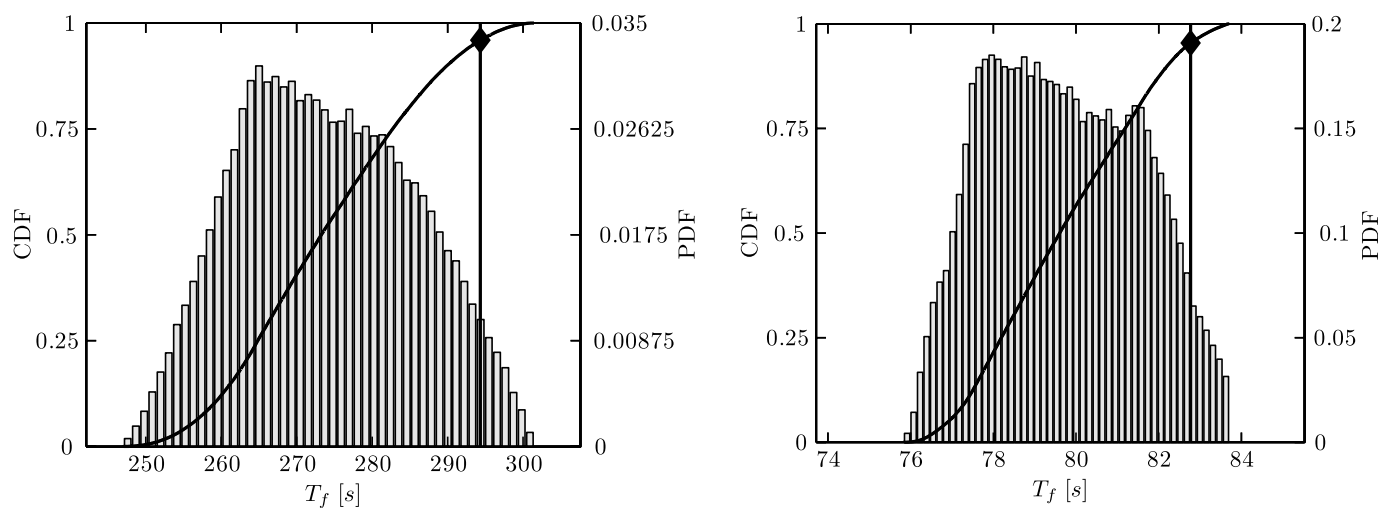

a) $M_{\infty}=9$

b) $M_{\infty}=10$
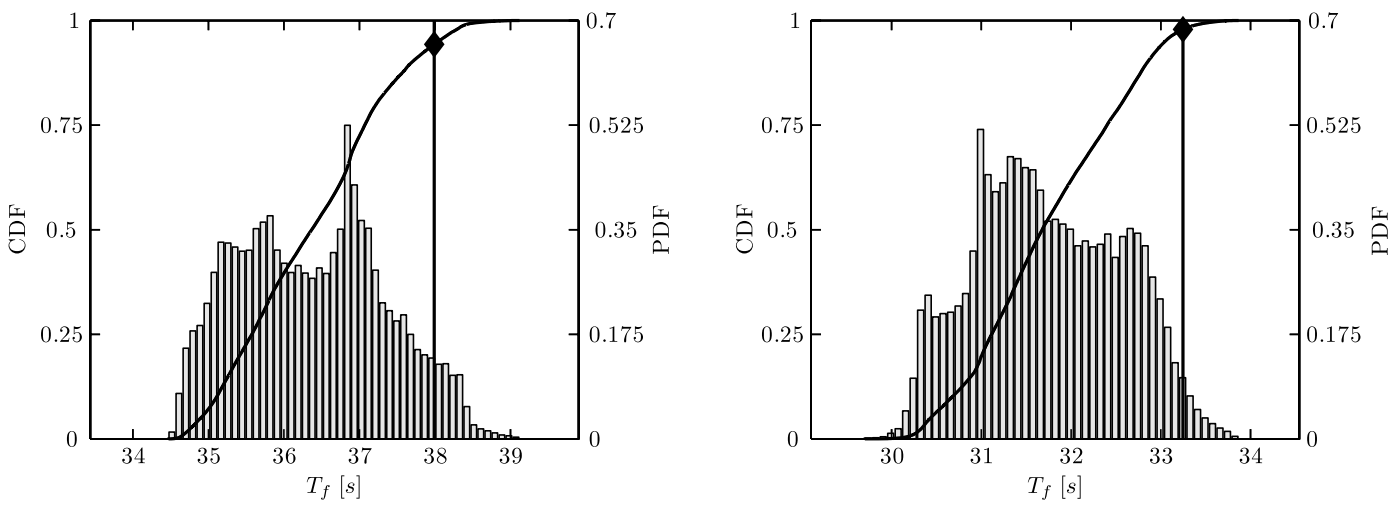

c) $M_{\infty}=11$

d) $M_{\infty}=12$

Fig. 16 PDF of the flight time for other Mach numbers (9 to 12). 
fit. These results indicate what happens when the stochastic variability is close to the error present in the deterministic analysis.

\section{Conclusions}

Stochastic collocation is an efficient approach for propagating uncertainty in aeroelastic and aerothermoelastic analyses. Reducedorder models used in hypersonic aeroelastic and aerothermoelastic analyses based on insufficient knowledge of pertinent physics associated with this class of problems require an uncertainty quantification approach. The results demonstrate that deterministic quantification of aeroelastic and aerothermoelastic stability boundaries may be inadequate for hypersonic vehicle analysis, and nondeterministic approaches may be required.

1) The effect of thermal loading on the aeroelastic stability of a typical section is estimated using the uncertainty propagation framework. The predicted flutter Mach number can decrease by up to $60 \%$. The deterministic flutter Mach number is close to the maximum flutter Mach number for each particular flight condition and elastic offset.

2) The probability distributions of the random inputs affect significantly the probabilistic results of the flutter Mach number and thus the probability of failure.

3) For the aerothermoelastic stability of a panel, the uncertainty inherent with turbulence modeling and transition prediction introduce additional sources of error. In this problem, uncertainties associated with transition location and the heat flux prediction influence the onset of instability. A 20\% variation range for the heat flux amplitude combined with the uncertainty in transition location yield to up to a $18 \%$ decrease in flight time. Note, however, that the transition is considered only before the leading edge of the panel and not on the panel itself.

\section{Acknowledgments}

This research is funded under NASA grant NNX08AB32A, with Donald Soloway and Jorge Bardina as technical monitors.

\section{Reference}

[1] McNamara, J. J., and Friedmann, P. P., "Aeroelastic and Aerothermoelastic Analysis in Hypersonic Flow: Past, Present, and Future," AIAA Journal, Vol. 49, No. 6, June 2011, pp. 1089-1122. doi:10.2514/1.J050882

[2] McNamara, J. J., Friedmann, P. P., Powell, K., Thuruthimattam, B., and Bartels, R., "Aeroelastic and Aerothermoelastic Behavior in Hypersonic Flow," AIAA Journal, Vol. 46, No. 10, Oct. 2008, pp. 2591-2610. doi:10.2514/1.36711

[3] Fidan, B., Mirmirani, M., and Ioannou, P. A., "Flight Dynamics and Control of Air-Breathing Hypersonic Vehicles: Review and New Directions," 12th AIAA International Space Planes and Hypersonic Systems and Technologies Conference, AIAA Paper 2003-7081, Dec. 2003

[4] Hallion, R. P., "The History of Hypersonics: Or, 'Back to the Future: Again and Again'," 43rd Aerospace Sciences Meeting and Exhibit, AIAA Paper 2005-329, Jan. 2005.

[5] Rodriguez, A. A., Dickeson, J. J., Cifdaloz, O., Kelkar, A., Vogel, J. M., and Soloway, D., "Modeling and Control of Scramjet-Powered Hypersonic Vehicles: Challenges, Trends, and Tradeoffs," AIAA Guidance, Navigation and Control Conference and Exhibit, AIAA Paper 2008-6793, Aug. 2008.

[6] Dolvin, D. J., "Hypersonic International Flight Research and Experimentation (HIFiRE), Fundamental Sciences and Technology Development Strategy," 15th AIAA International Space Planes and Hypersonic System and Technologies Conference, AIAA Paper 20082581, April-May 2008.

[7] Bertin, J. J., Hypersonic Aerothermodynamics, AIAA Education Series, AIAA, Washington, D.C., 1994, pp. 335-439.

[8] Anderson, J. D., Modern Compressible Flow with Historical Perspective, 3rd ed., McGraw-Hill, New York, 2004, pp. 585-644.

[9] Dugundji, J., and Calligeros, J. M., "Similarity Laws for Aerothermoelastic Testing," Journal of the Aerospace Sciences, Vol. 29, No. 8, Aug. 1962, pp. 935-950.

[10] Olejniczak, J., Candler, G. V., Wright, M. J., Hornung, H. G., and Leyva, I., "High Enthalpy Double-Wedge Experiments," 19th AIAA, Advanced
Measurement and Ground Testing Technology Conference, AIAA Paper 1996-2238, June 1996.

[11] Smith, V. K., Laster, M. L., and Boudreau, A. H., "Test and Evaluation Challenges for Hypersonic Air-Breathing Propulsion Systems," Proceedings of the Joint Test and Evaluation Meeting, AIAA Paper 1994-2675, 1994.

[12] Cox, C., Lewis, C., Pap, R., Golver, C., Priddy, K., Edwards, J., and McCarty, D., "Prediction of Unstart Phenomena in Hypersonic Aircraft," 6th AIAA International Aerospace Planes and Hypersonics Technologies Conference, AIAA Paper 1995-6018, April 1995.

[13] Culler, A., and McNamara, J. J., "Studies on Fluid-Thermal-Structural Coupling for Aerothermoelasticity in Hypersonic Flow," AIAA Journal, Vol. 48, No. 8, 2010, pp. 1721-1738. doi:10.2514/1.J050193

[14] Crowell, A., McNamara, J., and Miller, B., "Hypersonic Aerothermoelastic Response Prediction of Skin Panels Using Computational Fluid Dynamic Surrogates," Journal of Aeroelasticity and Structural Dynamics, Vol. 2, No. 2, 2011, pp. 3-30.

[15] Falkiewicz, N. J., Cesnik, C. E. S., Crowell, A. R., and Mcnamara, J. J., "Reduced-Order Aerothermoelastic Framework for Hypersonic Vehicle Control Simulation," AIAA Journal, Vol. 49, No. 8, 2011, pp. $1625-1646$. doi:10.2514/1.J050802

[16] Murugan, S., Harursampath, D., and Ganguli, R., "Material Uncertainty Propagation in Helicopter Nonlinear Aeroelastic Response and Vibration Analysis," AIAA Journal, Vol. 46, No. 9, Sept. 2008, pp. $2332-2344$ doi: $10.2514 / 1.35941$

[17] Beran, P. S., and Pettit, C. L., "A Direct Method for Quantifying Limit Cycle Oscillations Response Characteristics in the Presence of Uncertainties," 45th AIAA/ASME/ASCE/AHS/ASC Structures, Structural Dynamics, and Materials Conference, AIAA Paper 2004-1695, April 2004.

[18] Witteveen, J., Loeven, A., and Bijl, H., "An Adaptive Stochastic Finite Elements Approach Based on Newton-Cotes Quadrature in Simplex Elements," Computers \& Fluids, Vol. 38, No. 6, 2009, pp. 1270-1288. doi:10.1016/j.compfluid.2008.12.002

[19] Eldred, M. S., and Burkardt, J., "Comparison of Non-Intrusive Polynomials Chaos and Stochastic Collocation Methods for Uncertainty Quantification," 47th AIAA Aerospace Sciences Meeting, AIAA Paper 2009-976, Jan. 2009.

[20] Haldar, A., and Mahadevan, S., Probability, Reliability and Statistical Methods in Engineering Design, Wiley, New York, 2000, pp. 35-105.

[21] Ghanem, R. G., and Spanos, P. D., Stochastic Finite Elements: A Spectral Approach, Revised Edition, Springer-Verlag, New York, 1991, pp. 63-94.

[22] Mathelin, L., Hussaini, M. Y., and Zang, T. A., "Stochastic Approaches to Uncertainty Quantification in CFD Simulations," Numerical Algorithms Journal, Vol. 38, Nos. 1-3, March 2005, pp. 209-236. doi:10.1007/BF02810624

[23] Walters, R. W., "Towards Stochastic Fluid Mechanics via Polynomial Chaos," 41st Aerospace Sciences Meeting and Exhibit, AIAA Paper 2003-413, Jan. 2003.

[24] Eldred, M. S., Webster, C. G., and Constantine, P. G., "Design Under Uncertainty Employing Stochastic Expansion Methods," 12th AIAA/ ISSMO Multidisciplinary Analysis and Optimization Conference, AIAA Paper 2008-6001, Sept. 2008.

[25] Pettit, C., "Uncertainty Quantification in Aeroelasticity: Recent Results and Research Challenges," Journal of Aircraft, Vol. 41, No. 5, Sept.Oct. 2008, pp. 1217-1229.

[26] Hosder, S., Walters, R. W., and Balch, M., "Efficient Uncertainty Quantification Applied to the Aeroelastic Analysis of a Transonic Wing," 46th AIAA Aerospace Sciences Meeting and Exhibit, AIAA Paper 2008-729, Jan. 2008.

[27] Styuart, A. V., Livne, E., Demasi, L., and Mor, M., "Flutter Failure Risk Assessment for Damage-Tolerant Composite Aircraft Structures," AIAA Journal, Vol. 49, No. 3, March 2011, pp. 655-669. doi:10.2514/1.J050862

[28] Lindsley, N. J., Beran, P. S., and Pettit, C. L., "Integration of Model Reduction and Probabilistic Techniques with Deterministic MultiPhysics Models," 44th AIAA Aerospace Sciences Meeting and Exhibit, AIAA Paper 2006-192, Jan. 2006.

[29] Lindsley, N. J., Beran, P. S., and Pettit, C. L., "Effects of Uncertainty on Nonlinear Plate Response in Supersonic Flow," 9th AIAA/ISSMO Symposium on Multidisciplinay Analysis and Optimization, AIAA Paper 2002-5600, Sept. 2002

[30] Kurdi, M., Lindsley, N., and Beran, P., "Uncertainty Quantification of the Goland Wing's Flutter Boundary," AIAA Atmospheric Flight Mechanics Conference and Exhibit, AIAA Paper 2007-6309, Aug. 2007. 
[31] Choi, S.-K., Grandhi, R. V., Canfield, R. A., and Pettit, C. L., "Polynomial Chaos Expansion with Latin Hypercube Sampling for Estimating Response Variability," AIAA Journal, Vol. 42, No. 6 , June 2004, pp. 1191-1198. doi: $10.2514 / 1.2220$

[32] Golub, G. H., and Welsch, J. H., "Calculation of Gauss Quadrature Rules," Mathematics of Computation, Vol. 23, 1969, pp. 221-230. doi:10.1090/S0025-5718-69-99647-1

[33] Klimke, A., "Sparse Grid Interpolation Toolbox User's Guide, v5.1," Inst. for Applied Analysis and Numerical Simulation, Univ. of Stuttgart, Stuttgart, Germany, 2008.

[34] Bunjgartz, H.-J., and Dirnstorfer, S., "Multivariate Quadrature on Sparse Grids," Computing: Archives for Scientific Computing, Vol. 71, No. 1, 23 Aug. 2003, pp. 84-114.

[35] Sacks, J., Welch, W. J., Mitchell, T. J., and Wynn, H. P., "Design and Analysis of Computer Experiments," Statistical Science, Vol. 4, No. 4, 1989, pp. 409-435. doi:10.1214/ss/1177012413

[36] Jekabsons, G., "Adaptive Regression Splines Toolbox for Matlab," Ver. 1.3, Inst. of Applied Computer Systems, Riga Technical Univ., Riga, Latvia, Dec. 2009.

[37] Friedman, J. H., "Multivariate Adaptive Regression Splines," Annals of Statistics, Vol. 19, No. 1, March 1991, pp. 1-67.
[38] McNamara, J. J., and Friedmann, P. P., "Flutter Boundary Identification for Time-Domain Computational Aeroelasticity," AIAA Journal, Vol. 45, No. 7, July 2007, pp. 1546-1555. doi:10.2514/1.26706

[39] McNamara, J. J., "Aeroelastic and Aerothermoelastic Behavior of Two and Three Dimensional Lifting Surfaces in Hypersonic Flow," Ph.D. Thesis, Univ. of Michigan, Ann Arbor, MI, 2005.

[40] Shih, P., Prunty, J., and Mueller, R., "Thermostructural Concepts for Hypervelocity Vehicles," Journal of Aircraft, Vol. 28, No. 5, 1991, pp. 337-345. doi: $10.2514 / 3.46032$

[41] "5J-Metallic Materials and Elements for Aerospace Vehicle Structures," Department of Defense Handbook, Vol. 31, U.S. Dept. of Defense, Handbook MIL-HDBK, 2003.

[42] Eckert, E. R. G., "Engineering Relations for Heat Transfer and Friction in High-Velocity Laminar and Turbulent Boundary Layer Over Surfaces With Constant Pressure and Temperature," Transactions of the ASME, Vol. 78, No. 6, June 1956, pp. 1273-1283.

[43] Krist, S., Biedron, R., and Rumsey, C., "CFL3D User's Manual," Ver. 5.0, NASA TM-1998-208444, Sept. 1998

[44] "CFD++ User Manual, Turbulence Modeling," Ver. 10.1, Metacomp Technologies Inc., Agoura Hills, CA, 2010. 